\title{
Review: atypical antipsychotics may be useful in treating behavioural and psychological symptoms of dementia but cause adverse effects
}

Lee PE, Gill SS, Freedman M, et al. Atypical antipsychotic drugs in the treatment of behavioural and psychological symptoms of dementia: systematic review. BMJ 2004;329:75-8.

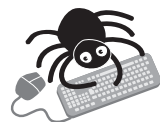

This article

contains extra text on the EBMH website

\section{Do atypical antipsychotic drugs attenuate behavioural and psychological symptoms of dementia in elderly people?}

\section{METHODS}

$\square$

Design: Systematic review.

Data sources: MEDLINE, EMBASE, and the Cochrane Library databases (searched to September 2003) plus hand searches and contact with experts.

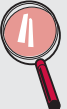

Study selection and analysis: Double blind randomised controlled trials assessing atypical antipsychotic drugs for behavioural and psychological symptoms of dementia (BPSD) (clozapine, risperidone, olanzapine, and quetiapine). Two reviewers independently assessed quality and abstracted information on participant demographics, duration, dosage, efficacy, and adverse events.

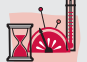

Outcomes: Efficacy was measured using the Behavioural Pathology in Alzheimer's Disease Score (BEHAVE-AD), the Cohen-Mansfield Agitation Inventory (CMAI) or the Neuropsychiatric Inventory - Nursing Home version (NPI-NH), or combinations of the above. Adverse events were noted, particularly extrapyramidal symptoms (Extrapyramidal Symptom Rating scale and the Simpson-Angus scale). Participant characteristics, study length, and dosage were also recorded.

\section{MAIN RESULTS}

Five trials met inclusion criteria $(n=1570$; mean age 82 years, $76 \%$ had Alzheimer's disease) from 77 reviewed abstracts. One RCT assessed olanzapine, four RCTs assessed risperidone. At 6-12 weeks, both risperidone and olanzapine significantly reduced scores for BPSD compared with placebo in four trials. One trial reported no significant difference on efficacy between two antipsychotics, risperidone (atypical) and haloperidol (typical). Atypical antipsychotics appeared to produce adverse events (extrapyramidal symptoms, somnolence, and abnormal gait). These results did not reach significance, except in the trial comparing risperidone and haloperidol, where extrapyramidal symptoms were more common with the typical antipsychotic haloperidol (see http://www. ebmentalhealth.com/supplemental for table).

\section{CONCLUSIONS}

Limited evidence exists for the efficacy of atypical antipsychotic drugs for BPSD, especially where compared with typical antipsychotics. Further trials are needed before atypical antipsychotics can be recommended for BPSD, including studies on adverse events

For correspondence: Philip Lee, pelee@providencehealth.bc.ca

Sources of funding: Eli Lilly Canada, Canadian Institutes of Health Research (CIHR), Saul A Silverman Family Foundation.

\section{Commentary}

$\Delta$ population based study of dementia found a point prevalence of psychopathology of over $60 \%$, and concluded that the lifetime risk approaches $90 \%{ }^{1}$ Such behavioural phenomena represent a huge public health burden, and we need evidence to define best practice in their treatment. The strengths of the paper by Lee et al lie in its thoughtful assessment of studies selected in a logical and transparent manner, although as a review it is not unique. ${ }^{2-4}$ It correctly highlights the uncertainty regarding whether changes in rating scale scores are clinically meaningful, the inherent limitations in extrapolating the results from brief trials to clinical practice, and the data regarding the liabilities of conventional antipsychotics, while cautioning against exuberant embrace of atypical antipsychotics.

The review, careful as it is, may have limited impact on practice. In merely promulgating the construct of "behavioural and psychological symptoms of dementia" it obscures the clinical complexity of the symptoms (and signs) that people experience, and ignores new efforts to treat specific symptoms and syndromes. If the target symptom-based approach were validated, say, for psychosis or aggression, clinicians would be more certain when, what, and how to treat. The paper dismisses conventional agents on the strength of a 1990 meta-analysis, in so doing ignoring at least six subsequent placebo controlled trials. More importantly, the rules for selecting studies understandably but unfortunately excluded at least eight placebo controlled trials of atypical agents in this population that have been presented publicly and are already influencing practice. This omission undercuts the conclusion that "only a few" randomised controlled trials have been conducted. The survey of safety and tolerability findings sometimes conflates statistically and clinically significant findings, so clinicians may not learn about the true likelihood of sedation or peripheral oedema with risperidone, for instance. At the same time, no guidance is given as to how to weigh the benefits against the risks of therapy in individual patients. By way of example, the paper accurately summarises new concerns about cerebrovascular adverse events apparently associated with risperidone and olanzapine use. However, if we do not use agents that have shown true (albeit modest) efficacy for serious agitation but have some liabilities, then what are we to do? Use agents without proven efficacy? Are other classes of agent superior? The authors fail to declare what treatment approach they would endorse; only what they would not.

Pierre N Tariot, MD

Professor of Psychiatry, Medicine, Neurology \& Aging and Developmental Biology, Director of Psychiatry at MCH, University of Rochester at MCH, Monroe Community Hospital, Rochester, NY, USA

1 Lyketsos CG, Sheppard JM, Steele CD, et al. Randomized, placebocontrolled, double-blind clinical trial of sertraline in the treatment of depression complicating Alzheimer's disease: initial results from the Depression in Alzheimer's Disease study. Am J Psychiatry 2000;157:1686-9.

2 Kindermann SS, Dolder CR, Bailey A, et al. Pharmacological treatment of psychosis and agitation in elderly patients with dementia: four decades of experience. Drugs Aging 2002:19:257-76.

3 Daiello LA, Beier MT, Poole Hoffman V, et al. Pharmacotherapy of behavioural and psychological symptoms of dementia; a review of atypical antipsychotics. Consultant Pharmacist 2003;18:2.

4 Belmonte DC, Streim JE. Safety profiles of atypical antipsychotics in the elderly. Curr Psychosis Ther Rep 2004;2:21-6. 